\title{
Nanoparticles in Endodontics - A Review
}

\author{
Pooja Ghanshyam Chandak ${ }^{1}$, Manoj Ghanshyamdasji Chandak², \\ Kajol Naresh Relan ${ }^{3}$, Madhulka Chandak ${ }^{4}$, Chanchal Rathi' ${ }^{5}$, Aditya Patel ${ }^{6}$
}

1, 2, 3, 4, 5, 6 Department of Conservative Dentistry and Endodontics, Sharad Pawar Dental College and Hospital, Datta Meghe Institute of Medical Sciences (Deemed to Be University), Sawangi Meghe, Wardha, Maharashtra, India.

\section{ABSTRACT}

\section{BACKGROUND}

The complex anatomy of root canal makes it very difficult to clean and shape to eliminate the bacteria in the canal. Multiple root canal medicaments and irrigants are used to eliminate the canal microbials. Many of the pathogens have undergone resistance to the medicaments and the irrigants used. Thus, antibacterial nanoparticles have been introduced to overcome this problem. Nano dentistry mainly infers the use of nanomaterials and dental nanorobots in the field of diagnosis and treatment, aiming improvement in complete oral health. The use of nanoparticles is extending in many oral health-related issues such as management of dentin hypersensitivity, elimination of bacterial biofilm, diagnosing as well as treating oral cancers, bone replacement and so on. It possesses a unique physical and chemical property. The usage of nanoparticles in different forms like solutions for irrigation, as intracanal medication, in conjunction with sealers or restorative materials aimed at evaluating and mainly improving the antimicrobial efficacy in endodontic and conservative treatments. These nanoparticles act on the bacteria through various mechanisms. The bactericidal effect of the nanoparticles has been investigated in many in vitro studies. In the field of endodontics, nanomaterials are inculcated mainly focusing on strategies improving antimicrobial efficacy, mechanical integrity of already diseased dentinal matrix, regeneration of the tissues and many more. Recently, many more new technologies are in process to be tested in the field, keeping an eye on overcoming the microbial challenge. This review intended to give a look on the literature available, uses, applications and advantages of the nanoparticles in endodontics.

\section{KEY WORDS}

Nanotechnology, Nanoparticles, Antibacterial Efficacy, Endodontics
Corresponding Author: Dr. Pooja Chandak, Sharad Pawar Dental College and Hospital, Datta Meghe Institute of Medical Sciences, Swangi Meghe, Wardha, Maharashtra, India.

E-mail: poojachandak1296@gmail.com

DOI: $10.14260 / \mathrm{jemds} / 2021 / 209$

How to Cite This Article: Chandak PG, Chandak MG, Relan KN, et al. Nanoparticles in endodontics - a review. $J$ Evolution Med Dent Sci 2021;10(13):976982, DOI: $10.14260 /$ jemds/2021/209

Submission 08-08-2020,

Peer Review 30-01-2021, Acceptance 06-02-2021, Published 29-03-2021.

Copyright (C) 2021 Pooja Ghanshyam Chandak et al. This is an open access article distributed under Creative Commons Attribution License [Attribution 4.0 International (CC BY 4.0)] 


\section{BACKGROUND}

Nanotechnology is a science that deals with development of new materials with new properties and functions through controlling and restructuring of the materials on a nano-meter scale of less than $100 \mathrm{~nm}$. The reference of Nanotechnology was initially given by Richard P. Feynman in the year 1960. The word NANO is a Greek word meaning DWARF. The concept of nanorobots was proposed by James Clerk Maxwell in 1867, and were called Maxwell's demons. Nanomaterial refers to the naturally occurring or synthetic material in an unbound state or an aggregate material with the particle size 1 - $100 \mathrm{~nm} \cdot .^{1-6}$ Nanomaterials are available in various size and shape. Nanomaterials have various properties like ultrasmall sizes, large surface area to mass ratio and increased chemical reactivity. 2,1,7,8 Nanoparticles have advantage of interacting with human body at subcellular and molecular level. This property leads to its high therapeutic efficacy and minimum side effects. ${ }^{9,10}$ Nanotechnology has various roles and biomedical applications like drug delivery, tissue regeneration, antimicrobial application, gene transfection and imaging. $4,1,10,11$ Nanotechnology was introduced to enhance complete oral health. This includes treatment of various oral problems like dentin hypersensitivity, biofilm removal, diagnosis and treatment of oral cancer etc. ${ }^{1}$ In endodontics it was introduced to improve antimicrobial efficacy, tissue regeneration and mechanical integrity of already affected dentin. ${ }^{1,12}$ Antibiotics are being used since a long time to eliminate and decrease the effect of microorganisms. But complete eradication of methicillin resistant Staphylococcus aureus and vancomycin-resistant streptococci have not been found. Such antibiotic resistant bacterias have characteristics like to defy the antimicrobial action of exchange of genetic materials, thickening of the peptidoglycan wall, or deficiency of the porin channels. This lead to increase in resistance of bacteria against antibiotics. To minimize this problem, the concept of endodontic nano particles has been introduced.

\section{CLASSIFICATION OF NANOPARTICLES}

1. On the basis of origin ${ }^{3}$
a. Natural
b. Artificial

2. On the basis of dimension ${ }^{3}$
a. Zero dimension - nano structures or nano particles
b. One dimension - nano rods
c. Two dimensional - thin films
d. Three dimensional - nano cones

3. On the basis of structural configuration ${ }^{3}$
a. Carbon based nano particles
b. Metal nano particles
c. Dendrimers
d. Composites

4. On the basis of composition ${ }^{1}$

a. Inorganic - Zinc oxide, iron oxide, titanium dioxide, cerium oxide, aluminum oxide, bioactive glass.

b. Metallic - Gold, silver, iron, copper, magnesium. c. Polymeric - Alginate, chitosan.

d. Quantum dots - Cadmium sulfide, cadmium selenide

e. Functionalised with drugs, photosensitizers, antibodies and proteins. These are the materials that have main body of one material and other particles or proteins bound on its surface or enclosed in it.

5. According to shape ${ }^{1}$
a. Particles
b. Spheres
c. Rods
d. Plates etc.

\section{ANTIBACTERIAL NANOPARTICLES}

Most common cause of pulpal and periradicular diseases is the microbial elements. Endodontic infection is polymicrobial in nature, just the difference occurs in organisms in primary or secondary infections. ${ }^{2}$ The infections begin in the form of biofilm. Ramchandran Nair discovered the pattern of microbial colonisation enclosed in the pulpal canal. ${ }^{2}$ Mahommad et al. defined microbial as a immobile polycellular community of microorganisms recognised by cells firmly attached to a surface and entangled in a matrix produced of extracellular polymeric substance on its own. ${ }^{2}$ The endodontic biofilm consists of $10-15 \%$ bacterial cells in $85-90 \%$ extracellular substance. ${ }^{2}$ Several factors enhance the virulence of these microorganisms.

Kishen in 2010 discussed the mechanism through which the endodontic microorganisms acquire resistance to the irrigants and medicaments used during treatment. These mechanisms were related to extracellular polymeric matrix, rate of bacterial growth, availability of nutrients and ability to adopt a resistant phenotype. ${ }^{2}$ The factor of resistance is bonding of biofilm structure to tooth surface through extracellular polymeric matrix. ${ }^{2}$ This provides mechanical stability to the biofilm. The core act as the nutritional spring. ${ }^{2}$ Increased bacterial virulence is due to difference in gene expression patterns in comparison to organisms found in a planktonic state. 2 This results in increased resistance of microbial biofilm against antibacterial agents.

Thus, nanoparticles as antimicrobial agents has been recently considered due to its enhanced antibacterial properties.

\section{NANOPARTICLE CONTRASTING BULK MATERIAL}

In comparison to bulk materials, nanoparticles are low solidity modicum with low coordination and unsatisfied bonds. This permits these particles to interconnect with other molecules easily. These particles exhibit quantum confinement effects. ${ }^{3}$ These particles exhibit high surface area to volume ratio. This takes part in determination of the energy stats of the molecules. ${ }^{3}$ Likely to bulk materials, nanoparticles disrupt the cell wall synthesis, but it also inhibits various enzymes like deoxyribonucleic acid (DNA)-dependent ribonucleic acid (RNA) polymerase and DNA gyrase. ${ }^{3}$ 


\section{ANTIBACTERIAL MECHANISM OF NANOPARTICLES}

The efficiency of bacterial elimination of nanoparticles is acquired by two different mechanisms.

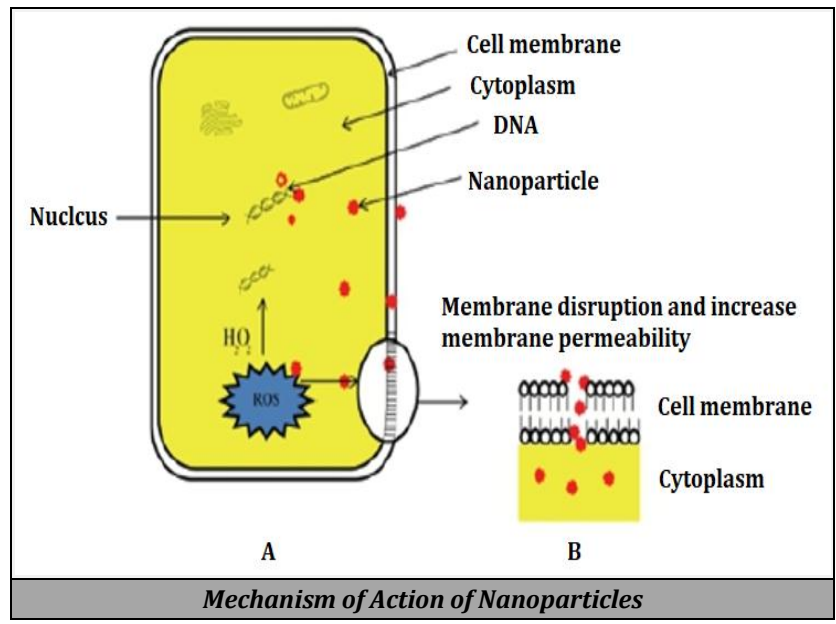

First Mechanism²

Nanoparticles bind to target bacterial cell membrane through electrostatic forces. This leads to change in the potential in membrane causing loss of polarity and loss of membrane rectitude. This disturbs the functions of microbial cell like respiration, transportation of nutrients and disturbance of energy transduction. All this process leads to cell death.

\section{Second Mechanism²}

Oxygen free radicals such as reactive-oxygen species (ROS), block the protein function and thus affect survival of the bacterial cell. It destroys DNA and results in over radical production.

Many other mechanisms ${ }^{3}$ have been discussed -

\section{Metal Ion Homeostasis}

Regulation of metabolic functions in microbes mainly metal ion homeostasis. Excess of metal-based NPs disturb this function which is necessary for life of cell that causes unrepairable destruction and plus retards the development or kill the bacteria.

\section{Protein and Enzyme Dysfunction}

Nanoparticles catalyse the amino acid side chain oxidation and form protein-bound carbonyls causing loss of catalytic action, polypeptide mortification and thus, passivation of crucial agitators.

\section{Generic Toxicity and Inhibition of Signal Transmission}

Nanoparticles interconnect with the biopolymers through their electrical configurations. It negatively influences the replication process of chromosome and plasmid DNA resulting in inhibition of signal transduction.

\section{Photokilling}

In the attendance of luminescence, photosensitive nanoparticles undergo photo-transformation of the cell layer and injure the polypeptides, particularly DNA which leads to photokilling of microbes. Photothermal killing is also reviewed for use in medical science.

\section{PROPERTIES OF NANOPARTICLES}

Introduction of nanoparticles against antimicrobial activity in treatment of oral cavity infections is possible because of its physicochemical properties, its antiadhesive nature, biocidal properties, potentially satisfying delivery capability. Nanoparticles have surface characteristics of interacting with plasma proteins restricting itself to reticuloendothelial system.

\section{Size}

Size of nanoparticles plays important role. The size is in $10-$ $100 \mathrm{~nm}$ range. Sizes less than $10 \mathrm{~nm}$ and greater than $100 \mathrm{~nm}$ does not possess therapeutic effect. Reasoning to very small particles, they are excreted out via kidneys and large molecules are occupied by the reticuloendothelial system for discarding.

\section{Surface Charge}

This property affects the bactericidal potency as specifically loaded NPs get simply bonded to opposite charged cell walls of the microbes, enhancing its outcome. A powerful charge has greater effectiveness but, compromise the stoutness of nanoparticle due to electric repellent forces among the nanoparticles. Thus, nanoparticles should be adequately loaded in context to negative or positive charging and amount of it.

\section{Surface Configuration}

Nanoparticles contain hydrophilic covering over its surface. These particles have high surface area / volume ratio. These properties improve its interaction with cellular receptors enhanced by binding ligand on the exterior of nanoparticles, thus these properties are responsible for its bio applications. This surface composition of nanoparticles have been made used by superparamagnetic iron oxide NPs.

\section{Protein Adsorption}

Polymers like polyethylene glycol, coated with NP prevent protein adsorption thus, increases their half-life.

\section{Functionalisation}

Raw NPs lack properties for biomedical applications. Hence, are amalgamated with various particles to alter its exterior structure. As a result of functionalisation, the NPs acts as a core and has coating of other materials over it. 


\section{SYNTHESIS OF NANOPARTICLES 3}

Approaches to prepare antibacterial nanoparticles -

1. Top-down approach: Reduces the size of the material from bulk to nanoscale utilising special treatments such as grinding / ablation / etching / sputtering.

2. Bottom-up approach: Preparation of NP / nanostructures utilising mostly chemical reactions.

Methods utilised in preparation include chemical, electrochemical, wet chemical, pyrolytic, microwave, hydrothermal, mycosynthesis, sonochemical, solution-gel, co-precipitation and biosynthesis (using plant extracts or microbial secretions).

\section{APPLICATIONS OF ANTIBACTERIAL NANOPARTICLES}

Antibacterial nanoparticles are used as drug delivery systems. It has applications in various fields of dentistry. In conservative dentistry it is used for restoration and curing of dentin hypersensitivity. It is also used to evolve the effect and properties of bonding agents like nano-adhesives and polymers. While in endodontics, it can be used as an endodontic irrigant against oral micro flora after adequate cleaning and shaping of the canals. ${ }^{3}$

\section{STRATEGIES FOR USE OF} ANTIBACTERIAL NANOPARTICLES

Antibacterial nanoparticles can be utilised alone or as a supplement. Nano based combinations have good penetration ability and slow down the release of active ingredients at desired site. The different strategies included are, ${ }^{3}$

- Using NP itself as a novel antibacterial agent.

- Using functionalised NP to provide therapeutic effects.

- Supplementation of antimicrobial photodynamic theory.

- Incorporation into other media such as root canal sealer such as EndoSequence BC Sealer and GuttaFlow.

\section{NANOPARTICLES AVAILABLE ${ }^{3}$}

\begin{tabular}{|cccc|}
\hline Organic & Non-Organic & Metals & Metal Oxides \\
$\begin{array}{c}\text { 1.Chitosan } \\
\text { nanoparticles }\end{array}$ & 1. Bioactive glass & 1. Gold & $\begin{array}{c}\text { 1. TiO2 } \\
\text { nanoparticles }\end{array}$ \\
& $\begin{array}{c}\text { 2. Bioactive mesoporous calcium } \\
\text { silicate NPs }\end{array}$ & 2. Silver & $\begin{array}{c}\text { 2. CuO } \\
\text { nanoparticles }\end{array}$ \\
$\begin{array}{c}\text { 2. Poly (lactic-co- } \\
\text { 00glycolic) acid }\end{array}$ & $\begin{array}{c}\text { 3. Tetracycline loaded } \\
\text { calcium-deficient hydroxyapatite } \\
\text { nanocarriers. }\end{array}$ & 3. Si & $\begin{array}{c}\text { 3. ZnO } \\
\text { nanoparticles }\end{array}$ \\
& Nanoparticles Available & $\begin{array}{c}\text { 4. MgO } \\
\text { nanoparticles }\end{array}$ \\
\hline
\end{tabular}

\section{ORGANIC NANOPARTICLES}

\section{Chitosan}

Chitosan (poly [1, 4-b-D-glucopyranose]) is a deacetylated derivative of chitin. ${ }^{1}$ It occurs in various configuration like powder (micro and nanoparticles), capsules, films, scaffolds, hydrogels, beads and bandages. ${ }^{1}$ Its structure is likely to matrix external to the cell and thus, is utilised to accelerate formation of connective tissue. It is a hydrophilic polymer. ${ }^{1}$ This nanoparticle is mainly introduced for bactericidal and administration of drug. It possesses bactericidal, antiviral and fungicidal effect. It has minimum inhibitory action at the range of $18-5000$ ppm. ${ }^{1}$ This depends on the organism, $\mathrm{pH}$, degree of deacetylation (DD), molecular weight, chemical modifications and presence of lipids and proteins. Chitosan nanoparticles (CS-NPs) in accordance to its electrostatic energy and diameter are anticipated to own improved bactericidal activity. ${ }^{1}$

\section{Mechanism of Action}

Chitosan exhibits contact mediated killing. ${ }^{1}$ This mechanism is due to electrical magnetism between positive chitosan and negative microbial cell sheet. This causes change in permeability of cell wall and rupture the cells and lead to percolation of the protein like and other intracellular components. ${ }^{1}$ In case of fungi, chitosan set foot in the nucleus, attach to deoxyribonucleic acid, and hinder ribonucleic acid and amino acid synthesis. ${ }^{1}$

\section{Application $^{1}$}

Chitosan nanoparticles in combination with zinc oxide eliminate biofilms. This occurs in a congregation and time dependent manner. The antibacterial properties are regained after aging for 90 days. This was evaluated by Kishen et al. Chitosan nanoparticles can be delivered in tubules of dentin of infected complex canals to improve disinfection of root canals. Biofilm bacteria provide efflux pumps for resistance to antimicrobials. In comparison to known effluence inhibitors, the antimicrobial efficiency of CS-NPs unaffected against microbial layer versus positively charged photosensitizers. Another confront in use of antimicrobial agents in the canal is the counter-balancing effect of various cell inactivators. Similarly, tissue for binders such as pulp and serum albumin prohibited the antimicrobial effect of CS-NPs significantly, whereas dentin, the dentin matrix, and lipopolysaccharides didn't influence the efficiency of CS-NPs. In another in vitro study, CS-NPs were used in combination with different brands of chlorhexidine to eliminate E. faecalis with potential application toward tissue regeneration using membrane barriers in periapical surgery. The addition of CS-NPs provided a significantly greater reduction of colony-forming units in agar plates as well as infected collagen membranes.

\section{Poly (Lactic-Co-Glycolic) Acid ${ }^{3}$}

Synergism effect of light and methylene blue-loaded NP in the reduction of bacterial counts in both planktonic phase and root canal has been found. It has been concluded that the use of poly (lactic-co-glycolic) acid NPs encapsulated with photoactive drugs might be a promising adjunct in antimicrobial endodontic treatment. 


\section{NON-ORGANIC NANOPARTICLES}

\section{Bioactive Glass}

Bioactive glass has osteoinductive effect and antibacterial properties. This material was introduced in the year $1971 .^{2}$ Bioactive glass consists of silicone dioxide, sodium oxide, calcium dioxide and phosphorus oxide (P205) at distinct agglomeration and depends on localised functional amendments for its antimicrobial effects. ${ }^{1}$

\section{Mechanism of Action ${ }^{2}$}

The action was shown to be through its ability to:

1. Release ions when in touch with a hydrous medium.

2. Increase the encompassing $\mathrm{pH}$.

3. Elevate the absorptive force around the microbial cell causing retardation of bacterial growth.

4. To precipitate calcium and phosphate ions in the bacterial cell membrane, disturbing its functions.

\section{Applications}

Zehnder et al. used amorphous nanometric bioactive glass (BAG), $20-60 \mathrm{~nm}$ in size. ${ }^{1}$ In vitro study concluded less antibacterial effect of BAG in comparison to calcium hydroxide. $45 \mathrm{~S} 5$ bioactive glass nanoparticles produced good effects against $E$. faecalis than micro-sized bioactive glass particles. ${ }^{1}$ Planktonic bacteria were killed in a better manner. The reduced antibacterial efficacy of nano-BAG may be due to 10 -fold increased leaching out of silica and rise in $\mathrm{pH}$ of solution by greater than 3 units by the latter. ${ }^{1}$

The contradictory results on BAG microparticles and nanoparticles on antibacterial efficacy demands further research before clinical application. ${ }^{1}$

\section{Bioactive Mesoporous Calcium Silicate NPs ${ }^{3}$}

These NPs are $100 \mathrm{~nm}$ in size. It has greater surface area and larger pore volume.

\section{Properties}

- Injectability

- Apatite mineralisation

- Osteo stimulation

- Drug delivery

- Antibacterial efficiencies.

\section{Tetracycline-Loaded Calcium-Deficient Hydroxyapatite 3}

It is osteoconductive drug delivery system. It is assembled from apatite nanocarriers having ability to provide sustained release of tetracycline in the periodontium.

\section{METALLIC NANOPARTICLES}

\section{Gold $^{3}$}

Mechanism

- NPs attached to membrane, changes the membrane potential and decreases ATP level.

- Inhibits transfer ribonucleic acid (tRNA) binding to the ribosome.

\section{Properties}

- Non-toxic

- Functionalisation

- Multifunctional effects

- $\quad$ Easy to detect.

- Photothermal activity.

\section{Silver}

Silver compounds as NPs are used due to their antibacterial property. Silver in metallic state appears inert. In presence of dampness it appears in ionized form. ${ }^{3}$ The silver ions are greatly interactive. The ions attach to tissue proteins, and cause alteration in tissue. The ions form nuclear membrane blockade and result in death of cell. Problems with use of silver NPs are the blackening of dentin and toxic effect on mammalian cells. ${ }^{3}$

\section{Mechanism of Action ${ }^{1}$}

Silver acts on multiple targets and produces antibacterial effect. The particles sequentially interact with sulfhydryl groups of proteins and DNA, altering hydrogen bonding, unwinding of DNA and finally interfere with cell-wall synthesis. Silver nanoparticles (Ag-NPs) disturbs the microbial cell sheet and increase penetrability. This causes outflow of cell contents.

\section{Applications $^{1}$}

In a study by Hiraishi et al. found the effect of silver diamine fluoride against $E$. faecalis biofilms. ${ }^{1}$ Complete eradication of microbial layer after an hour of interaction was seen. In another study, $0.02 \%$ Ag-NP gel was used as intracanal medication for 7 days. It had better result in E. faecalis biofilm destruction, in comparison to calcium hydroxide and syringe irrigation with higher concentration Ag-NP $(0.1 \%)$ solution ${ }^{1}$. Ag-NPs of $9 \mathrm{~nm}$ size showed the lowest minimal inhibitory concentration on planktonic E. faecalis as compared with sodium hypochlorite and chlorhexidine. Ag-NPs are not harmful to fibroblast cells. Ag-NP-loaded mesoporous bioactive glass (Ag-MBG) when investigated on E. faecalis biofilms in pulpal canals showed significant destruction of the biofilm. It should be limitedly used as medicament than that as an irrigant.

\section{SILICONE}

\section{Properties}

- Influence functions like differentiation, adhesion and spreading

- Nontoxicity

- Stability

\section{METAL OXIDES}

\section{Zinc Oxide Nanoparticles ${ }^{2}$}

Zinc oxide nanoparticles have high antibacterial effectiveness. Its high $\mathrm{pH}$ environment destroys microbial cells. Zinc oxide nanoparticles increase permeability of the cell wall, leach out cytoplasmic content and cause destruction of cell. The 
bactericidal effect is directly related to size of the particles. Smaller the size, higher is the antibacterial effect. Zinc oxide NP dissociates zinc ions in the microbial cell and disturb its adjunctive action resulting in further damage. Its antibacterial effect depends on its concentration. Higher the levels maximum is the antibacterial effect.

Kishan et al. assessed antibacterial and antibiofilm efficiency of zinc oxide (ZO) nanoparticles on E. faecalis. It showed that these nanoparticles reduce the formation of number of colonies of $E$. faecalis in a layer. $95 \%$ reduction was seen in adherence of E. faecalis in dentin. Another study showed reduced thickness of the E. faecalis biofilm after 3 days of contact with ZO nanoparticles. Study concluded that ZO NP deleted E. faecalis in a planktonic state, but not in a biofilm state. Zinc oxide nanoparticles when added to polyethylene glycol showed varying effects against $P$. aeruginosa, E. faecalis, C. albicans, S. aureus and Kocuria rhizophila. However, further studies are recommended if antibacterial effects have to be improved and more in vivo evaluation is also required.

\section{Titanium Dioxide ${ }^{3}$}

Mode of Action

- $\quad$ Oxidative stress via the generation of ROS

- Lipid peroxidation that causes to enhance membrane fluidity and disrupt cell integrity.

\section{Properties}

- Photocatalytic properties

- Stable

- Effective fungicidal for fluconazole-resistant strains.

\begin{abstract}
Magnesium Oxide and Calcium Oxide ${ }^{2}$
Nanoparticles consisting magnesium were put forward for use as antibacterial vehicle in opposition to endodontic pathogens due to their proven antimicrobial effects over gram-positive and gram-negative bacteria, spores and viruses. Magnesiumcontaining nanoparticles are either magnesium-oxide nanoparticles or magnesium halogen-containing nanoparticles such as chlorine, bromine and fluorine. Nanoparticle containing combination of magnesium and halogen enters inside the bacterial cell membrane, and disturbs the membrane potential. This causes DNA binding and lipid peroxidation effects causing more damage to bacterial cell. Magnesium-oxide nanoparticles were found to be bactericidal when present in a hydrous configuration as a result of activity of superoxide anions established on the microbial cell surface. Monzavi et al. studied bactericidal effect of discrete combinations of $\mathrm{MgO}$ nanoparticles ( $5 \mathrm{mg} / \mathrm{L}$ and $10 \mathrm{mg} / \mathrm{L})$ and $5.25 \%$ sodium hypochlorite $(\mathrm{NaOCl})$ and $2 \%$ chlorhexidine (CHX) against endodontic pathogens such as $E$. faecalis, $S$. aureus and Candida albicans. No significant dissimilarity was seen in the bactericidal effect of the irrigant solutions used against the tested endodontic pathogens. However, the incorporation of magnesium oxide nanoparticles in an irrigant solution produced improved antibacterial activity in comparison to sodium hypochlorite.
\end{abstract}

\section{Copper Oxide Nanoparticles ${ }^{3}$}

Mode of action is by crossing of NPs from the bacterial cell membrane and then damaging the vital enzymes of bacteria.

\section{Properties}

- Effective against gram-positive and gram-negative bacteria

- High stability

- Antifungal activity

\section{NANOPARTICLES IN SEALERS AND RESTORATIVE MATERIALS}

Obturating material should 3-dimensionally lock the assembled canal space to prevent microbial reinvasion. The addition of NPs in sealers and restorative materials is to acquire decreased microbial invasion, enhanced bactericidal action, enhanced substantivity of sealers, and enhanced dispersion of antimicrobial activity. ${ }^{1}$ Nanocomposites are recently invented by incorporation of nanoparticles in it. These composites have good high initial polish and polish retention properties, and enhanced mechanical properties and handling characteristics. ${ }^{5}$ The addition of CS-NP and zinc oxide nanoparticles in a zinc oxide-based root canal sealer and resin-based root canal sealer increased bactericidal effect. ${ }^{1}$ DaSilva et al. used CS-NP incorporated zinc oxide-eugenol sealer in taurine root canals in his ex vivo study. It was found that nanoparticle modified sealer prevented biofilm formation. ${ }^{1}$

BAGNP has showed to encourage termination of the intermediate gap between canal enclosure and obturating substance. ${ }^{1}$ Incorporating bioactive glass particles into the polymers, rendered composite bioactive, increased its initial locking ability. Polyisoprene and polycaprolactone composites with bioactive glass demonstrated good outcome in one rooted canal. Quaternary ammonium polyethyleneimine nanoparticles (QPEINPs) improved the antibacterial efficacy of various materials. ${ }^{1}$ Barros et al. added QPEI-NPs into AH Plus (Dentsply International, York, PA) and Pulp Canal Sealer EWT (SybronEndo, Orange, CA). This enhanced the wetting ability and external loading of the sealers. Beyth et al. and Kesler Shvero et al. found that incorporation of QPEI-NPs in 2paste epoxy-amine resin sealer attracted $E$. faecalis to exterior and led to tissue destruction. This prevented bacterial growth. ${ }^{1}$ QPEI-NPs showed antibacterial action by adsorbing and penetrating inside the wall of microbial cell, interacting with the polypeptide and lipid layer in the cell layer and thus, preventing the interchange of important ions, weakening of the cell wall and damaging the cell.

Most NPs showed contact-mediated and time-dependent antibacterial activity. Thus, their incorporation into materials enhanced the bactericidal effect by discouragement of microbial biofilm development on resin-dentin interface. The ex vivo studies on NPs stresses on thorough and systematised studies toward possible views of clinical application.

\section{CONCLUSIONS}

Nanoparticle-based treatments have the ability to enhance bactericidal effect in endodontics. Functionalised nanoparticles via surface modifications would provide a chance to administer drug to infection area to specifically interconnect with cell layer and microbes. ${ }^{1}$ New compositions 
of nanoparticles are being introduced on the basis of clinical requirements. The idea of nanoparticles in medical science and dentistry should be given importance for future developments.

Financial or other competing interests: None.

Disclosure forms provided by the authors are available with the full text of this article at jemds.com.

\section{REFERENCES}

[1] Shrestha A, Kishen A. Antibacterial nanoparticles in endodontics: a review. J Endodon 2016;42(10):1417-26.

[2] Ibrahim AIO, Moodley DS, Petrik L, et al. Use of antibacterial nanoparticles in endodontics. SADJ 2017;72(3):105-12.

[3] Bhushan J, Maini C. Nanoparticles: a promising novel adjunct for dentistry. Indian J Dent Sci 2019;11(3):16773.

[4] Asiri A, Inamuddin, Mohammad A, eds. Nanotechnology in Dentistry. In: Applications of Nanocomposite materials in dentistry. Woodhead Publishing Series in Biomaterials 2019:191-203.
[5] Panchbhai A. Nanocomposites: past, present and future of dentistry. In: Applications of Nanocomposite Materials in Dentistry. Woodhead Publishing 2019:181-90.

[6] Kishen A. Nanotechnology in endodontics: current and potential clinical applications. Switzerland: Cham, Springer International Publishing 2015.

[7] Cohen ML. Nanotubes, nanoscience and nanotechnology. Materials Science and Engineering C 2001;15(1-2):1-11.

[8] Thomas JP, Peppas N, Sato M, et al. Nanotechnology and biomaterials. Boca Raton, FL: CRC Taylor and Francis 2006.

[9] Curtis A, Wilkinson C. Nantotechniques and approaches in biotechnology. Trends in Biotechnol 2001;19(3):97-101.

[10] Venugopal J, Prabhakaran MP, Low S, et al. Nanotechnology for nanomedicine and delivery of drugs. Curr Pharm Des 2008;14(22):2184-200.

[11] Cushing BL, Kolesnichenko VL, O'connor CJ. Recent advances in the liquid-phase syntheses of inorganic nanoparticles. Chem Rev 2004;104(9):3893-946.

[12] Costerton JW, Lewandowski Z, DeBeer D, et al. Biofilms, the customized microniche. J Bacteriol 1994;176(8):2137-42. 